\title{
The hidden role of NLRP3 inflammasome in diabesity-related COVID-19 exacerbations: lessons for drug repurposing
}

\author{
Ilaria Bertocchi ${ }^{1}$, Federica Foglietta ${ }^{2}$, Debora Collotta ${ }^{1}$, Carola Eva ${ }^{1}$, Vincenzo \\ Brancaleone $^{3}$, Chris Thiemermann ${ }^{4}$, and Massimo Collino ${ }^{1}$ \\ ${ }^{1}$ University of Turin \\ ${ }^{2}$ Universita degli Studi di Torino Scuola di Scienze della Natura \\ ${ }^{3}$ University of Basilicata \\ ${ }^{4}$ Queen Mary University of London Faculty of Medicine and Dentistry
}

June 26, 2020

\begin{abstract}
COVID-19, the illness caused by SARS-CoV-2, has a wide-ranging clinical spectrum that, in the worst-case scenario, involves a rapid progression to severe acute respiratory syndrome and even death. Epidemiological data show that "diabesity", the association of obesity and diabetes, is among the main risk factors associated with high morbidity and mortality. The increased susceptibility to SARS-CoV-2 infection documented in diabesity argues for initial defects in defense mechanisms, most likely due to an elevated systemic metabolic inflammation ("metaflammation"). The NLRP3 inflammasome is a master regulator of metaflammation and has a pivotal role in the pathophysiology of diabesity. Here we discuss the most recent findings suggesting contribution of NLRP3 inflammasome to the increase in complications in COVID-19 patients with diabesity. We also review current pharmacological strategies for COVID-19, focusing on treatments whose efficacy could be due, at least in part, to interference with the activation of the NLRP3 inflammasome.
\end{abstract}

\begin{abstract}
COVID-19, the illness caused by SARS-CoV-2, has a wide-ranging clinical spectrum that, in the worst-case scenario, involves a rapid progression to severe acute respiratory syndrome and even death. Epidemiological data show that "diabesity", the association of obesity and diabetes, is among the main risk factors associated with high morbidity and mortality. The increased susceptibility to SARS-CoV-2 infection documented in diabesity argues for initial defects in defense mechanisms, most likely due to an elevated systemic metabolic inflammation ("metaflammation"). The NLRP3 inflammasome is a master regulator of metaflammation and has a pivotal role in the pathophysiology of diabesity. Here we discuss the most recent findings suggesting contribution of NLRP3 inflammasome to the increase in complications in COVID-19 patients with diabesity. We also review current pharmacological strategies for COVID-19, focusing on treatments whose efficacy could be due, at least in part, to interference with the activation of the NLRP3 inflammasome.
\end{abstract}

\section{Metaflammation in "diabesity" and COVID-19}

The severe acute respiratory syndrome coronavirus 2 (SARS-CoV-2) is a novel member of human betacoronavirus (mutational ssRNA viruses) causing the disease named COVID-19 (for coronavirus disease 2019), which is recognized as a pandemic on March 11, 2020 by the World Health Organization (WHO). COVID-19 is a syndrome with a wide clinical spectrum: it can be asymptomatic or, in the majority of cases, cause mild symptoms that are indistinguishable from other respiratory infections, but it may also lead to a rapid progression to severe acute respiratory syndrome (SARS), with respiratory failure and even death (Zhou et al., 2020). 
The activation of signalling receptors of the innate immune system is the first step of the physiological response to virus infection. However, this innate immune system activation, when excessive, may evoke hyperinflammation and tissue damage in patients with severe COVID-19, making it an important aspect of the pathophysiology of this syndrome (Netea et al., 2020).

Older people are more likely to suffer from the most serious complications of SARS-CoV-2 infection: this is likely due to the physiological decrease in the efficacy of the immune system and the simultaneous increase in age-related inflammatory response termed 'inflammaging' (Latz \& Duewell, 2018). Moreover, aging and the modern lifestyle typical of Western societies are both associated with an increase in comorbidities, such as "diabesity", the association of obesity and diabetes, which defines a combination of primarily metabolic disorders evoked by impairment of both lipids and sugars metabolisms (Potenza, Nacci, De Salvia, Sgarra, Collino \& Montagnani, 2017; Tschop \& DiMarchi, 2012). The close relationship existing between impaired metabolism and immunity in diabesity is well documented, and the term "metaflammation" has been coined to indicate this pathophysiologic inflammatory response derived by metabolic alterations and dysfunctions (Hotamisligil, 2017; Mastrocola, Aragno, Alloatti, Collino, Penna \& Pagliaro, 2018). An increasing number of studies report that patients with severe obesity or type 2 diabetes mellitus (T2DM) exhibit an increased circulating concentration of proinflammatory cytokines, such as IL- $1 \beta$, IL- 6 and tumor necrosis factor (TNF)$\alpha$ (Huang et al., 2020). The metaflammation, induced in response to excess calories intake in the adipose tissue, may subsequently involve other organs such as the skeletal muscle, liver, heart and lung, leading to metabolic and cardiovascular alterations.

Compared with people of a normal weight, obese individuals have an increased susceptibility to develop chronic diseases and infections (Huttunen \& Syrjanen, 2010; Milner \& Beck, 2012; Wolowczuk et al., 2008). Patients with T2DM developing COVID-19 are twice as likely to require ventilation and intensive care unit (ICU) supports and their mortality is 3-fold higher than in non-diabetic COVID-19 patients (Guan et al., 2020; Yang et al., 2020). Obesity carries a 5-fold increase in risk of developing severe pneumonia from COVID-19 (Cai et al., 2020) and diabesity is a well-recognised risk factor for severe infections (Almond, Edwards, Barclay \& Johnston, 2013; Huttunen \& Syrjanen, 2013), sleep apnoea (Dixon \& Peters, 2018), poor immune response and scant outcomes in patients with respiratory disease (Green \& Beck, 2017). According to United States data, 37\% of 3,615 hospitalized COVID-19 cases were obese, and the obesity increased the odds of ICU admission (Lighter et al., 2020). In a French study enrolling COVID-19 patients admitted to the ICU, the requirement for ventilation was increased by 7 -fold in patients with a body mass index (BMI) $>35 \mathrm{~kg} / \mathrm{m}^{2}$, and this was independent of age and presence of diabetes or hypertension (Simonnet et al., 2020). Adipocytes express angiotensin-converting enzyme-2 (ACE2), which is the receptor for SARS-CoV-2 (Yan, Zhang, Li, Xia, Guo \& Zhou, 2020) and, like in human and simian immunodeficiency virus (HIV/SIV) infections, adipose tissue may act as a reservoir for SARS-CoV-2 (Jia et al., 2020; Wanjalla et al., 2019). As widely documented (Huttunen \& Syrjanen, 2013; Singh, Gupta, Ghosh \& Misra, 2020), obese people and individuals with T2DM are more likely to develop a dysfunctional immune response that provokes viral infection and makes failed the elimination of the pathogen. Specific reasons for this are not still understood, even if one reasonable explanation could be an excessive metaflammation, often due to an over-activation of specific inflammatory pathways, including, but not limited to, the NLRP3 inflammasome.

\section{The NLRP3 inflammasome and its role in viral infection}

NLRP3 inflammasome is a large, multimeric protein complex assembled within the cell upon the recognition of unique molecular patterns by a germline-encoded pattern recognition receptor (PRR) (Yang, Wang, Kouadir, Song \& Shi, 2019). Among the PRRs known to form inflammasomes, NLRP3 belongs to the family containing both a nucleotide-binding oligomerization domain (NOD) and leucine-rich repeat (LRR)containing proteins. NLRP3 is expressed in many cell types, as innate immunity, endothelial, hematopoietic, lung epithelial, kidney and cardiac cells (Ratajczak \& Kucia, 2020). We and others have previously demonstrated that NLRP3 inflammasome overactivation contributes to the pathogenesis of cardio-metabolic disorders (Mastrocola et al., 2016; O'Riordan et al., 2019; Wang et al., 2020; Zuurbier et al., 2019), whereas NLRP3 deficiency results in reduced systemic inflammation, along with decreased immune cell activation 
and improved insulin resistance (Grant \& Dixit, 2013; Vandanmagsar et al., 2011). NLRP3 inflammasome is also a crucial player in immune defence of the host against many pathogens, including viruses (Xu et al., 2020; Zhao \& Zhao, 2020). Once activated, its amino-terminal pyrin domain (PYD) is able to recruit the downstream adaptor protein apoptosis-associated speck-like protein containing a caspase-recruitment domain (ASC) and to assemble the scaffold complex important to recruit the inflammasome effector, procaspase-1, which becomes activated. Active form of caspase- 1 clivates both pro-IL-1 $\beta$ and pro-IL-18 into their biologically active forms (Dinarello, 2009). Caspase-1 can also activate another substrate, gasdermin D (GSDMD), that is capable to mediate pyroptosis by creating pore channels in cell membranes. Pyropoptosis is a lytic form of necrosis used by the innate immune system to disrupt pathogen replication and intracellular accumulation, thanks to the formation of pore-induced intracellular traps (Jorgensen, Zhang, Krantz \& Miao, 2016). Despite its role in facilitating pathogen clearance, inflammasome activation can also be detrimental to the host by enhancing viral dissemination (Lupfer, Malik \& Kanneganti, 2015). To date, it is still unclear whether SARS-CoV-2 activates the NLRP3 inflammasome (Yap, Moriyama \& Iwasaki, 2020), but this is likely, as SARS-CoV also activates the NLRP3 inflammasome by modulating either ion channel activity or ASC ubiquitination. Moreover, after binding to SARS-CoV-2, ACE2 is internalized, leading to high cytosolic levels of angiotensin II, which is known to act as an activator of the NLRP3 inflammasome in lung, kidney cells and cardiomyocytes (Pinar, Scott, Huuskes, Tapia Caceres, Kemp-Harper \& Samuel, 2020; Sun et al., 2017; Zhao et al., 2018). At the same time, given that caspase-1 affects expression of several innate immunity genes and viral replication (Bauer et al., 2012), the NLRP3 inflammasome may alter cellular physiology and homeostasis also modulating gene expression, leading to altered innate antiviral defense pathways and impacting therefore on host responsiveness to pathogens like SARS-CoV-2.

As diabesity is a high-risk factor for both influenza infections and hospitalization for respiratory illness during seasonal influenza (Karlsson, Sheridan \& Beck, 2010; Kwong, Campitelli \& Rosella, 2011), we may speculate that a chronic over-activation of the NLRP3 inflammasome, due to metabolic impairments, may contribute to alterations in the innate immune response to viral infection, making it more permissive and severe (Figure $1)$.

\section{The NLRP3 inflammasome and its involvement in the cytokine storm}

The activation of both innate and adaptive immunity after recognition of viral antigens produces a large amount of pro-inflammatory cytokines and chemokines even in moderate cases of COVID-19. In some patients this activation is exacerbated, leading to a 'cytokine storm', which in turn causes severe lung injury, multiple organ failure and death (Sarzi-Puttini et al., 2020; Xu et al., 2020). The mechanisms by which SARS-CoV-2 subverts the body's innate antiviral cytokine responses are yet to be elucidated. Nevertheless, most recent research on SARS-CoV suggests that pyroptosis may have a pivotal role. Pyroptosis is a form of programmed cell death with an high inflammatory scenario that is commonly observed with cytopathic viruses (Fink \& Cookson, 2005). As mentioned before, NLRP3 inflammasome regulates pyroptosis by GSDMD-mediated membrane rupture along with spontaneous release of cytosolic contents into the extracellular spaces. The activation of pyroptosis in alveolar macrophages and in recruited monocyte-derived macrophages by SARS-CoV-2 aggravates pneumonia and ARDS. The release of alarmins, including viral particles, ATP, reactive oxygen species (ROS) as well as cytokines, chemokines and lactate dehydrogenase $(\mathrm{LDH})$, elicits an immediate reaction from surrounding immune cells, inducing a pyroptotic chain reaction. Different studies have reported elevated levels of LDH, a cytosolic enzyme that is measured for monitoring pyroptosis (Rayamajhi, Zhang \& Miao, 2013), in patients with the severe form of the disease and worse outcomes (Lippi, Mattiuzzi, Bovo \& Plebani, 2020). In keeping with this and other observations, we can suggest the NLRP3 inflammasome-mediated pyroptosis as one of the key mechanisms involved inCOVID-19 (Figure 1). In accordance, a further exacerbation of symptoms may derive from the pyroptosis induced release of viral RNA and antigens that, by reaching the circulation, may generate immune complex and depositions in other target organs, initiating severe inflammatory cascades. Hence, in addition to local damage, cytokine storm also has ripple effects across the body, evoking organ damage and multi-organ failure (Ruan, Yang, Wang, Jiang \& Song, 2020). 


\section{NLRP3 inflammasome and resolution of inflammation}

Resolution of inflammation is an active process triggered by the onset of inflammation itself (Perretti \& D'Acquisto, 2009). The main actors of this process consist of specialized proresolving mediators (SPMs), whose list is constantly updated, including annexin A1 (AnxA1), formyl-peptide receptor 2 (FPR2) and lipoxin $\mathrm{A}_{4}\left(\mathrm{LXA}_{4}\right)$ (Brancaleone et al., 2013; Dufton et al., 2010; Norling, Dalli, Flower, Serhan \& Perretti, 2012; Serhan, Chiang \& Dalli, 2015). Very recently, AnxA1 has been reported to be involved in the regulation of NLRP3 inflammasome function (Galvao et al., 2020), independently from the binding to its cognate receptor FPR2 (Galvao et al., 2020). Indeed, lower levels of AnxA1 are associated to an increased activation of NLRP3, thus raising IL-1 $\beta$ releasing (Kelley, Jeltema, Duan \& He, 2019). In addition, AnxA1 is known to trigger resolution pathway by engaging FPR2 (Brancaleone, Dalli, Bena, Flower, Cirino \& Perretti, 2011; Perretti \& D'Acquisto, 2009) and (Machado et al., 2020)AnxA1/FPR2 signaling axis has been identified to control and limit viral infection (Alessi, Cenac, Si-Tahar \& Riteau, 2017; Lopategi et al., 2019). Therefore, impairment of this axis could unbalance the immune response to infections, thus generating an ineffective response which in turn could lead to increased cytokine storm and exacerbation of COVID-19 symptoms.

Does NLRP3 inflammasome activation drive the exacerbation of inflammation and disease pathology in obese/diabetic patients with COVID-19?

The specific reasons for the higher susceptibility of obese and diabetic patients to SARS-CoV2 are unclear, but chronic exposure to a low grade NLRP3 inflammasome-dependent metaflammation may well be a key driver. Diet-induced alterations in the gut microbiome and related increased gut leakiness of bacterial wall lipopolysaccharides (endotoxins) are known to promote organs and tissues NLRP3 inflammasome activation by Toll-like receptor (TLR) pathways. This event is followed by the accumulation of the cytokines of the IL-1 family, which are known to modulate the insulin producing pancreatic ?-cell function (Tack, Stienstra, Joosten \& Netea, 2012). We and others have demonstrated that NLRP3 inflammasome overactivation is involved not only in the pathogenesis of diabesity, but also in the exacerbation of related cardiovascular injuries, including myocardial infarction, by increasing the local inflammatory response and, at the same time, decreasing the efficiency of endogenous protective responses (Mastrocola et al., 2016; Xu et al., 2013). NLRP3 inflammasome activation is involved in endothelial lysosome membrane permeabilization, cathepsin B release, impaired glycocalyx thickness (Ikonomidis et al., 2019), thus further contributing to the enhanced susceptibility to cardiovascular injury. Similarly, the diabesity-related basal activation of the NLRP3 inflammasome cascade, leading to increase in either gastrointestinal and vascular permeability, may contribute to exacerbate SARS-CoV-2 systemic diffusion and enhance the intricate mechanisms of intracellular cross-talk operational in the pathogenesis of COVID-19.

\section{The NLRP3 inflammasome and pharmacologic treatments for COVID-19}

Till date, there are no specific medications available to treat COVID-19. Clinical trials are in process on several drugs, mainly based on the drug repurposing approach to redevelop a compound/drug for the use in a different disease (COVID-19) other than that of its original use. This review summarizes recent documentations on clinically approved drugs, repurposed to counteract COVID-19 infection, whose potential efficacy can be due, at least in part, to interference with the NLRP3 inflammasome cascade (Figure 2).

We systematically searched the PubMed and Google Scholar databases, clinical trials.gov, chictr.org.cn/searchprojen.aspx and https://www.clinicaltrialsregister.eu until June 25th, 2020 to prepare this section of the narrative review on the role of NLRP3 inflammasome in drugs repurposed in COVID-19. We also accessed the full text of the relevant cross references from the search results.

\section{Anti-IL-1 agents}

There are at least 8 clinical trials on pharmacological strategies specifically aimed to target the main product of NLRP3 inflammasome activation, the cytokine IL-1 $\beta$. They include studies on the human anti-IL-1 $\beta$ monoclonal antibody Canakinumab (ClinicalTrials.gov identifier: NCT04362813, NCT04365153, NCT04348448) and the recombinant non-glycosylated human IL-1 receptor antagonist 
Anakinra (NCT04324021, NCT04330638, NCT0432402, NCT04357366 and NCT04339712). Besides, several other clinical trials are evaluating the effects of drugs targeting IL-6, whose serum concentration are drastically increased in patients with NLRP3 inflammasome-mediated condition (Abbate, Toldo, Marchetti, Kron, Van Tassell \& Dinarello, 2020). A cross-talk mechanism linking IL-1 $\beta$ to IL-6 has been documented. IL-1 $\beta$ induces IL-6 gene transcriptionvia a PI-3-kinase/AKT-dependent pathways (Cahill \& Rogers, 2008) and, at the same time, blockade of IL-6 signalling blunts the activation of NLRP3 inflammasome (Powell et al., 2015; Wu et al., 2018). Thus, drugs able to interfere with IL-6 receptor activation may inhibit NLRP3dependent IL-1 $\beta$ release and, in a similar way, anti-IL-1 $\beta$ strategies significantly reduce IL-6 release, with an effective control of the cytokine-storm. Several clinical trials have been approved to test the efficacy of Sarilumab (NCT04315298 and NCT04327388, EudraCT identifier: 2020-001162-12 and 2020-001390-76) and Tocilicizumab (ChinaXiv identifier: 202003.00026 and 20000.30894), two humanized monoclonal antibodies against the IL-6 receptor, thus confirming that targeting cellular machineries leading to cytokine overproduction, such as the NLRP3 inflammasome, holds promise for COVID-19 therapy.

\section{Antimalarial agents}

Chloroquine (CQ) and its analogue hydroxychloroquine (HCQ), two well-known anti-malarial drugs, have been proposed for severe COVID-19 treatment, with more than 70 clinical trials registered on clinicaltrials.gov. Several studies have demonstrated the anti-inflammatory activity of chloroquine mainly due to significant inhibition ofcytokines release, including IL-1 $\beta$, from monocytes and macrophages (Hong et al., 2004). Recently, a direct role for chloroquine in inhibiting the expression of NLRP3 inflammasome components has been demonstrated (Chen, Wang, Zhu, Lu, Liu \& Zheng, 2017). Besides, hydroxychloroquine exerts its anti-inflammatory effect by inhibiting cathepsin-mediated NLRP3 inflammasome activation (Tang et al., 2018).

\section{Antiviral agents}

The administration of the protease inhibitor ritonavir in association with lopinavir, has been tested for the treatment of SARS-CoV-2 infection and COVID-19 pneumonia (Cao et al., 2020) and there are several on-going clinical trials in China (NCT04255017, NCT04261907, NCT04286503, NCT04295551), Hong Kong (NCT04276688), Republic of Korea (NCT04307693) and in Europe (NCT04315948, NCT04328285). The protease inhibitor darunavir is under investigation as an alternative drug to lopinavir/ritonavir treatment in COVID-19 (NCT04252274 and ChiCTR2000029541). Other two clinical trials (NCT04261270 and NCT04303299) have been proposed to evaluate oseltamivir, a neuraminidase inhibitor approved for the treatment of influenza, alone or in combination with ritonavir or favipiravir, darunavir and chloroquine. Interestingly, the three antiviral drugs here mentioned interfere with NLRP3 inflammasome activation. Ritonavir has been demonstrated to inhibit caspase-1 activation leading to reduced cleavage of pro-IL-18 (Lopez-Castejon \& Pelegrin, 2012), whereas darunavir limits the expression of key regulators of NLRP3 inflammasome complex formation, TLR4 and NF- $x \mathrm{~B}$ (Zhang et al., 2018). The interaction of oseltamivir with NLRP3 inflammasome has been demonstrated in an in vivo study on influenza A pdm09 virus infection (Jia et al., 2018).

\section{Interferons}

Interferons (IFNs) are under investigation for the treatment of COVID-19, mainly in combination with lopinavir/ritonavir (Chinese Clinical Trial Registry identifier: ChiCTR2000029387) or lopinavir/ritonavir/ribavirin (NCT04276688 and EudraCT database identifier: 2020-001023-14).

Selective interactions between type I-IFNs and NLRP3 inflammasome have been documented in the literature. For instance, both type-I IFNs and IFN- $\gamma$ evoke higher expression of inducible nitric oxide (NO) synthase, increasing levels of endogenous NO, which inhibits NLRP3 oligomerization by means of direct S-nitrosylation of the NLRP3 protein, avoiding full inflammasome organization (Mishra et al., 2013). Another mechanism resides in the ability of type-I IFN to decrease the NLRP3 inflammasome activation via the signal transducer and activator of transcription 1 (STAT1) that induces caspase- 1 to process the IL- $1 \beta$ precursor (Kopitar-Jerala, 2017). 


\section{Antibiotics}

Few clinical trials are recruiting patients with COVID-19 to test the efficacy of the known macrolide antibiotic azithromycin alone (NCT04332107, NCT04381962, NCT04369365, NCT04371107) or in combination with hydroxychloroquine (NCT04339816, and NCT04336332). The lipophilic antibiotics tetracyclines have also been proposed as treatment for COVID-19 (Sodhi \& Etminan, 2020), with just one clinical study (NCT04371952) that is recruiting COVID-19 patients to test the efficacy and safety of the tetracycline doxycycline.

The beneficial effects of azithromycin against lipopolysaccharides (LPS)-induced pulmonary neutrophilia have been documented to involve NLRP3 inflammasome inhibition (Bosnar et al., 2011; Gualdoni, Lingscheid, Schmetterer, Hennig, Steinberger \& Zlabinger, 2015), which is also involved in mediating tetracycline beneficial effects in experimental models of diabetic retinopathy and ischemic stroke (Chen et al., 2017; Lu, Xiao \& Luo, 2016).

\section{Immunosuppressants}

Three clinical trials are recruiting COVID-19 patients to test the efficacy of the immunosuppressant sirolimus(NCT04341675, NCT04371640 and NCT04374903), whereas no clinical study on the calcineurin inhibitor cyclosporine in COVID-19 has been recorded, despite it has been hypothesized as potential candidate for SARS-CoV-2 infection (Cure, Kucuk \& Cumhur Cure, 2020). Both the immunosuppressants can interfere with NLRP3 inflammasome. Sirolimus inhibits NLRP3 inflammasome activation in macrophages through autophagy induction (Ko, Yoon, Lee \& Oh, 2017), whereas cyclosporine targets cyclophilin D to avoid the opening of mitochondria permeability transition pore (MPT), which is strictly involved in NLRP3 inflammasome activation (Iyer et al., 2013).

Another well-known NLRP3 inhibitor included in several clinical protocols for COVID-19 patients is the classical anti-mitotic drug colchicine, with at least ten ongoing clinical trials (NCT04328480, NCT04322682, 2020-001841-38, NCT04392141, 2020-001511-25, NCT04322565, NCT04375202, NCT04360980, 2020-00160316, NCT04363437) and 3 others ready to start (NCT04367168, NCT04355143, NCT04326790). Several mechanisms have been proposed to explain how colchicine can suppress NLRP3 inflammasome activation. First, colchicine can inhibit the expression of pyrin gene, avoiding the assembly of the NLRP3 inflammasome complex (Nidorf, Eikelboom \& Thompson, 2014). Second, colchicine inhibits activation of caspase-1 and mature IL-1 $\beta$ (Otani et al., 2016). Third, colchicine inhibits P2X7-induced pore formation, a key step in NLRP3 inflammasome activation following ATP exposure (Marques-da-Silva, Chaves, Castro, Coutinho-Silva \& Guimaraes, 2011).

Other interesting small molecules with immunomodulatory properties, already proposed for COVID-19 infection and with documented modulatory activity on NLRP3 inflammasome, are the Janus kinase (JAK) inhibitor baricitinib and the Bruton's tyrosine kinase (BTK) inhibitor acalabrutinib. A study on baricitinib has already been concluded with interesting results (NCT04358614) and others are recruiting patients for both the treatments (Baricitinib: NCT04320277 and NCT04390464; Acalabrutinib: NCT04346199 and NCT04380688). Experimental evidence for key roles of the JAK and BTK cascades in either activation of the NLRP3 inflammasome and diabesity pathogenesis have been reported in the literature (Collotta et al., 2020; Furuya et al., 2018)

Up to nowadays six clinical trials (NCT04347980, NCT04325061, NCT04395105, NCT04344730, NCT04360876, NCT04327401), reported on clinicaltrials.gov are recruiting patients to test the efficacy of the corticosteroid dexamethasone, whose beneficial effects in airway inflammation has been recently demonstrated to involve lung inhibition of the activity of NLRP3 inflammasome and the release of IL-1 $\beta$ and IL-18 (Guan, Ma, Fan, Chen, Miao \& Wu, 2020).

\section{Conclusions.}

Both infection and individual dysfunctional immune response elicited by SARS-CoV-2 have been reported to contribute to COVID-19 pathogenesis. Thus, it is more than a speculation that controlling the inflammatory 
response may be as important as targeting the virus. The evidences here reported suggest a potential role for the NLRP3 inflammasome pathway as cross-talk mechanism involved in both cellular events. Indeed, pharmacological targeting of NLRP3 inflammasome could be a feasible approach to counteract the pathology at multiple levels, ranging from the interference with viral infection to the reassessment of unbalanced immune responses. In addition, patients with exacerbated disease might reveal an impaired resolution process, which could again reflect on NLRP3 hyper-activation contributing to the "cytokine storm". Interestingly, some of the pharmacological strategies under investigation for COVID-19 therapy include drugs able to directly disrupt the NLRP3 inflammasome cascade or to affect the transcriptional activity of factors involved in the synthesis of the main components of the NLRP3 inflammasome complex. Further studies are needed to better elucidate the pathophysiological involvement of NLRP3 inflammasome in this specific context and to prove the efficacy and safety of related pharmacological strategies. Overall, the findings here reviewed underlie intriguing perspective within the intricate mechanisms involved in the COVID-19 pathogenesis, and, thus, they may contribute to raise hope for discovering new strategies to battle one of the main pandemic of our modern times.

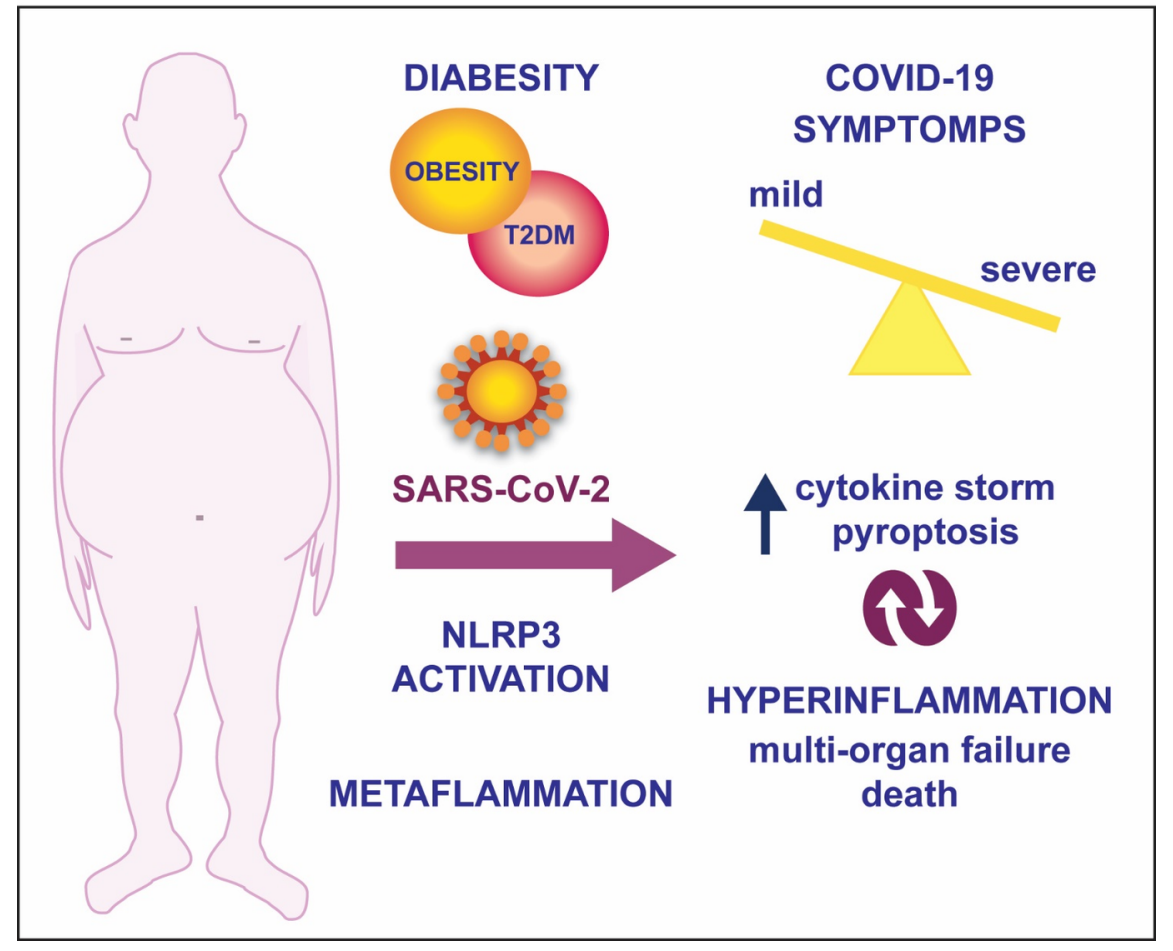

Figure 1. The figure schematizes the relationship between diabesity- the association of obesity and diabetesand SARS-CoV-2 infection response. Diabesity increases the susceptibility to exacerbation of COVID-19 throughout an excessive metabolic inflammatory response (metaflammation) that is regulated, at least in part, by the NLRP3 inflammasome complex. NLRP3 is also a master regulator of the cytokine storm and pyroptosis, both evoked by SARS-CoV-2 infection. 


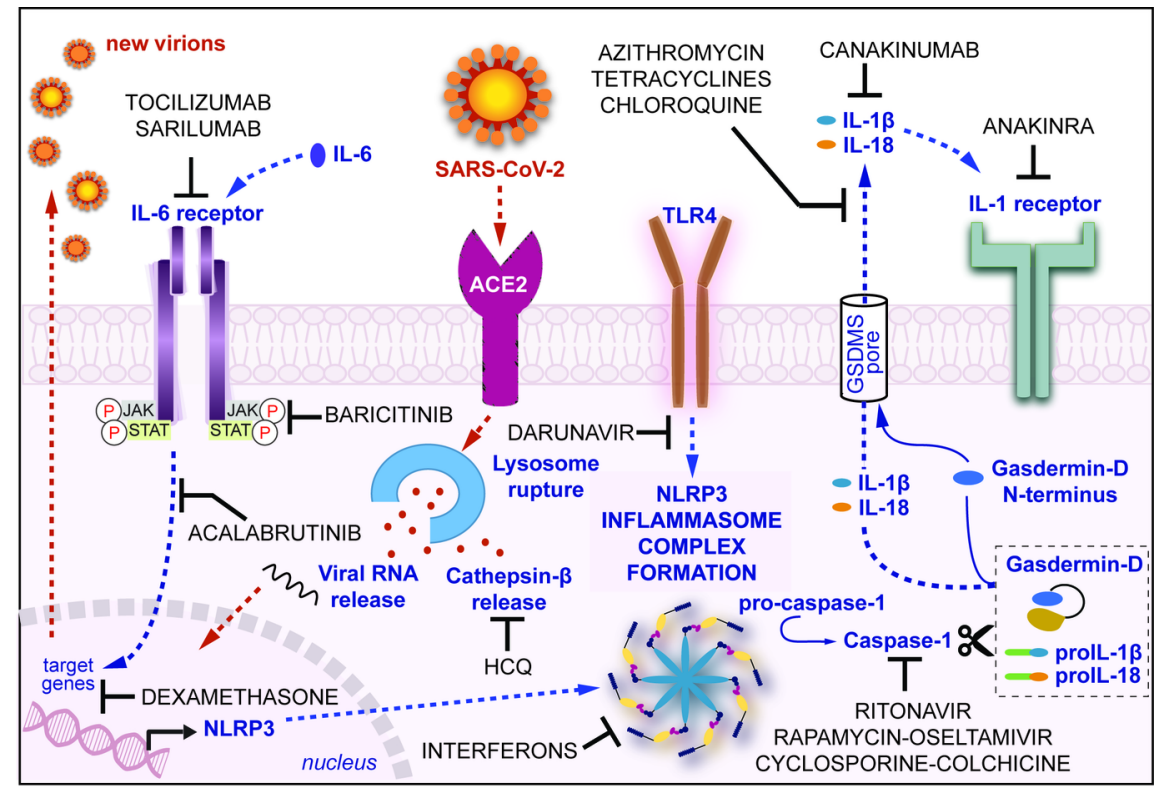

Figure 2. A schematic illustration of plausible NLRP3 inflammasome-related mechanisms targeted by clinically approved drugs, repurposed to manage COVID-19. Following SARS-CoV-2 interaction with ACE2 receptors, NLRP3 inflammasome is up-regulated to induce immune response against the virus. Overall, the over-activation of NLRP3 amplifies the innate immune response and contributes to cell death by pyroptosis and virus propagation. HCQ: Hydroxychloroquine.

\section{References}

Abbate A, Toldo S, Marchetti C, Kron J, Van Tassell BW, \& Dinarello CA (2020). Interleukin-1 and the Inflammasome as Therapeutic Targets in Cardiovascular Disease. Circ Res 126: 1260-1280.

Alessi MC, Cenac N, Si-Tahar M, \& Riteau B (2017). FPR2: A Novel Promising Target for the Treatment of Influenza. Front Microbiol 8: 1719.

Almond MH, Edwards MR, Barclay WS, \& Johnston SL (2013). Obesity and susceptibility to severe outcomes following respiratory viral infection. Thorax 68: 684-686.

Bauer RN, Brighton LE, Mueller L, Xiang Z, Rager JE, Fry RC, et al. (2012). Influenza enhances caspase-1 in bronchial epithelial cells from asthmatic volunteers and is associated with pathogenesis. J Allergy Clin Immunol 130: 958-967 e914.

Bosnar M, Cuzic S, Bosnjak B, Nujic K, Ergovic G, Marjanovic N, et al. (2011). Azithromycin inhibits macrophage interleukin-1beta production through inhibition of activator protein-1 in lipopolysaccharideinduced murine pulmonary neutrophilia. Int Immunopharmacol 11: 424-434.

Brancaleone V, Dalli J, Bena S, Flower RJ, Cirino G, \& Perretti M (2011). Evidence for an anti-inflammatory loop centered on polymorphonuclear leukocyte formyl peptide receptor 2/lipoxin A4 receptor and operative in the inflamed microvasculature. J Immunol 186: 4905-4914.

Brancaleone V, Gobbetti T, Cenac N, le Faouder P, Colom B, Flower RJ, et al. (2013). A vasculo-protective circuit centered on lipoxin A4 and aspirin-triggered 15-epi-lipoxin A4 operative in murine microcirculation. Blood 122: 608-617. 
Cahill CM, \& Rogers JT (2008). Interleukin (IL) 1beta induction of IL-6 is mediated by a novel phosphatidylinositol 3-kinase-dependent AKT/IkappaB kinase alpha pathway targeting activator protein-1. J Biol Chem 283: 25900-25912.

Cai Q, Chen F, Wang T, Luo F, Liu X, Wu Q, et al. (2020). Obesity and COVID-19 Severity in a Designated Hospital in Shenzhen, China. Diabetes Care.

Cao B, Wang Y, Wen D, Liu W, Wang J, Fan G, et al. (2020). A Trial of Lopinavir-Ritonavir in Adults Hospitalized with Severe Covid-19. N Engl J Med 382: 1787-1799.

Chen W, Zhao M, Zhao S, Lu Q, Ni L, Zou C, et al. (2017). Activation of the TXNIP/NLRP3 inflammasome pathway contributes to inflammation in diabetic retinopathy: a novel inhibitory effect of minocycline. Inflamm Res 66: 157-166.

Chen X, Wang N, Zhu Y, Lu Y, Liu X, \& Zheng J (2017). The Antimalarial Chloroquine Suppresses LPS-Induced NLRP3 Inflammasome Activation and Confers Protection against Murine Endotoxic Shock. Mediators Inflamm 2017: 6543237.

Collotta D, Hull W, Mastrocola R, Chiazza F, Cento AS, Murphy C, et al. (2020). Baricitinib counteracts metaflammation, thus protecting against diet-induced metabolic abnormalities in mice. Mol Metab 39: 101009 .

Cure E, Kucuk A, \& Cumhur Cure M (2020). Cyclosporine therapy in cytokine storm due to coronavirus disease 2019 (COVID-19). Rheumatol Int 40: 1177-1179.

Dinarello CA (2009). Immunological and inflammatory functions of the interleukin-1 family. Annu Rev Immunol 27: 519-550.

Dixon AE, \& Peters U (2018). The effect of obesity on lung function. Expert Rev Respir Med 12: 755-767.

Dufton N, Hannon R, Brancaleone V, Dalli J, Patel HB, Gray M, et al. (2010). Anti-inflammatory role of the murine formyl-peptide receptor 2: ligand-specific effects on leukocyte responses and experimental inflammation. J Immunol 184: 2611-2619.

Fink SL, \& Cookson BT (2005). Apoptosis, pyroptosis, and necrosis: mechanistic description of dead and dying eukaryotic cells. Infect Immun 73: 1907-1916.

Furuya MY, Asano T, Sumichika Y, Sato S, Kobayashi H, Watanabe H, et al. (2018). Tofacitinib inhibits granulocyte-macrophage colony-stimulating factor-induced NLRP3 inflammasome activation in human neutrophils. Arthritis Res Ther 20: 196.

Galvao I, de Carvalho RVH, Vago JP, Silva ALN, Carvalho TG, Antunes MM, et al. (2020). The role of annexin A1 in the modulation of the NLRP3 inflammasome. Immunology 160: 78-89.

Grant RW, \& Dixit VD (2013). Mechanisms of disease: inflammasome activation and the development of type 2 diabetes. Front Immunol 4: 50.

Green WD, \& Beck MA (2017). Obesity Impairs the Adaptive Immune Response to Influenza Virus. Ann Am Thorac Soc 14: S406-S409.

Gualdoni GA, Lingscheid T, Schmetterer KG, Hennig A, Steinberger P, \& Zlabinger GJ (2015). Azithromycin inhibits IL-1 secretion and non-canonical inflammasome activation. Sci Rep 5: 12016.

Guan M, Ma H, Fan X, Chen X, Miao M, \& Wu H (2020). Dexamethasone alleviate allergic airway inflammation in mice by inhibiting the activation of NLRP3 inflammasome. Int Immunopharmacol 78:106017.

Guan WJ, Ni ZY, Hu Y, Liang WH, Ou CQ, He JX, et al. (2020). Clinical Characteristics of Coronavirus Disease 2019 in China. N Engl J Med 382: 1708-1720. 
Hong Z, Jiang Z, Liangxi W, Guofu D, Ping L, Yongling L, et al.(2004). Chloroquine protects mice from challenge with CpG ODN and LPS by decreasing proinflammatory cytokine release. Int Immunopharmacol 4: $223-234$.

Hotamisligil GS (2017). Inflammation, metaflammation and immunometabolic disorders. Nature 542: 177185.

Huang C, Wang Y, Li X, Ren L, Zhao J, Hu Y, et al. (2020). Clinical features of patients infected with 2019 novel coronavirus in Wuhan, China. Lancet 395: 497-506.

Huttunen R, \& Syrjanen J (2010). Obesity and the outcome of infection. Lancet Infect Dis 10: 442-443.

Huttunen R, \& Syrjanen J (2013). Obesity and the risk and outcome of infection. Int J Obes (Lond) 37: 333-340.

Ikonomidis I, Pavlidis G, Katsimbri P, Andreadou I, Triantafyllidi H, Tsoumani M, et al. (2019). Differential effects of inhibition of interleukin 1 and 6 on myocardial, coronary and vascular function. Clin Res Cardiol 108: 1093-1101.

Iyer SS, He Q, Janczy JR, Elliott EI, Zhong Z, Olivier AK, et al.(2013). Mitochondrial cardiolipin is required for Nlrp3 inflammasome activation. Immunity 39: 311-323.

Jia X, Liu B, Bao L, Lv Q, Li F, Li H, et al. (2018). Delayed oseltamivir plus sirolimus treatment attenuates H1N1 virus-induced severe lung injury correlated with repressed NLRP3 inflammasome activation and inflammatory cell infiltration. PLoS Pathog 14:e1007428.

Jia X, Yin C, Lu S, Chen Y, Liu Q, Bai J, et al. (2020). Two Things about COVID-19 Might Need Attention. Preprints.

Jorgensen I, Zhang Y, Krantz BA, \& Miao EA (2016). Pyroptosis triggers pore-induced intracellular traps (PITs) that capture bacteria and lead to their clearance by efferocytosis. J Exp Med 213: 2113-2128.

Karlsson EA, Sheridan PA, \& Beck MA (2010). Diet-induced obesity impairs the T cell memory response to influenza virus infection. J Immunol 184: 3127-3133.

Kelley N, Jeltema D, Duan Y, \& He Y (2019). The NLRP3 Inflammasome: An Overview of Mechanisms of Activation and Regulation. Int J Mol Sci 20.

Ko JH, Yoon SO, Lee HJ, \& Oh JY (2017). Rapamycin regulates macrophage activation by inhibiting NLRP3 inflammasome-p38 MAPK-NFkappaB pathways in autophagy- and p62-dependent manners. Oncotarget 8:40817-40831.

Kopitar-Jerala N (2017). The Role of Interferons in Inflammation and Inflammasome Activation. Front Immunol 8: 873 .

Kwong JC, Campitelli MA, \& Rosella LC (2011). Obesity and respiratory hospitalizations during influenza seasons in Ontario, Canada: a cohort study. Clin Infect Dis 53: 413-421.

Latz E, \& Duewell P (2018). NLRP3 inflammasome activation in inflammaging. Semin Immunol 40: 61-73.

Lighter J, Phillips M, Hochman S, Sterling S, Johnson D, Francois F, et al. (2020). Obesity in patients younger than 60 years is a risk factor for Covid-19 hospital admission.

. Clin Infect Dis

Lippi G, Mattiuzzi C, Bovo C, \& Plebani M (2020). Current laboratory diagnostics of coronavirus disease 2019 (COVID-19). Acta Biomed 91: 137-145.

Lopategi A, Flores-Costa R, Rius B, Lopez-Vicario C, Alcaraz-Quiles J, Titos E, et al. (2019). Frontline Science: Specialized proresolving lipid mediators inhibit the priming and activation of the macrophage NLRP3 inflammasome. J Leukoc Biol 105: 25-36. 
Lopez-Castejon G, \& Pelegrin P (2012). Current status of inflammasome blockers as anti-inflammatory drugs. Expert Opin Investig Drugs 21: 995-1007.

Lu Y, Xiao G, \& Luo W (2016). Minocycline Suppresses NLRP3 Inflammasome Activation in Experimental Ischemic Stroke. Neuroimmunomodulation 23: 230-238.

Lupfer C, Malik A, \& Kanneganti TD (2015). Inflammasome control of viral infection. Curr Opin Virol 12: $38-46$.

Machado MG, Tavares LP, Souza GVS, Queiroz-Junior CM, Ascencao FR, Lopes ME, et al. (2020). The Annexin A1/FPR2 pathway controls the inflammatory response and bacterial dissemination in experimental pneumococcal pneumonia. FASEB J 34: 2749-2764.

Marques-da-Silva C, Chaves MM, Castro NG, Coutinho-Silva R, \& Guimaraes MZ (2011). Colchicine inhibits cationic dye uptake induced by ATP in P2X2 and P2X7 receptor-expressing cells: implications for its therapeutic action. Br J Pharmacol 163: 912-926.

Mastrocola R, Aragno M, Alloatti G, Collino M, Penna C, \& Pagliaro P (2018). Metaflammation: TissueSpecific Alterations of the NLRP3 Inflammasome Platform in Metabolic Syndrome. Curr Med Chem 25:12941310.

Mastrocola R, Collino M, Penna C, Nigro D, Chiazza F, Fracasso V, et al. (2016). Maladaptive Modulations of NLRP3 Inflammasome and Cardioprotective Pathways Are Involved in Diet-Induced Exacerbation of Myocardial Ischemia/Reperfusion Injury in Mice. Oxid Med Cell Longev 2016: 3480637.

Milner JJ, \& Beck MA (2012). The impact of obesity on the immune response to infection. Proc Nutr Soc 71: 298-306.

Mishra BB, Rathinam VA, Martens GW, Martinot AJ, Kornfeld H, Fitzgerald KA, et al. (2013). Nitric oxide controls the immunopathology of tuberculosis by inhibiting NLRP3 inflammasome-dependent processing of IL-1beta. Nat Immunol 14: 52-60.

Netea MG, Giamarellos-Bourboulis EJ, Dominguez-Andres J, Curtis N, van Crevel R, van de Veerdonk FL, et al. (2020). Trained Immunity: a Tool for Reducing Susceptibility to and the Severity of SARS-CoV-2 Infection. Cell 181: 969-977.

Nidorf SM, Eikelboom JW, \& Thompson PL (2014). Targeting cholesterol crystal-induced inflammation for the secondary prevention of cardiovascular disease. J Cardiovasc Pharmacol Ther 19: 45-52.

Norling LV, Dalli J, Flower RJ, Serhan CN, \& Perretti M (2012). Resolvin D1 limits polymorphonuclear leukocyte recruitment to inflammatory loci: receptor-dependent actions. Arterioscler Thromb Vasc Biol 32: 1970-1978.

O'Riordan CE, Purvis GSD, Collotta D, Chiazza F, Wissuwa B, Al Zoubi S, et al. (2019). Bruton's Tyrosine Kinase Inhibition Attenuates the Cardiac Dysfunction Caused by Cecal Ligation and Puncture in Mice. Front Immunol 10: 2129.

Otani K, Watanabe T, Shimada S, Takeda S, Itani S, Higashimori A, et al. (2016). Colchicine prevents NSAID-induced small intestinal injury by inhibiting activation of the NLRP3 inflammasome. Sci Rep 6: 32587.

Perretti M, \& D'Acquisto F (2009). Annexin A1 and glucocorticoids as effectors of the resolution of inflammation. Nat Rev Immunol 9:62-70.

Pinar AA, Scott TE, Huuskes BM, Tapia Caceres FE, Kemp-Harper BK, \& Samuel CS (2020). Targeting the NLRP3 inflammasome to treat cardiovascular fibrosis. Pharmacol Ther 209: 107511.

Potenza MA, Nacci C, De Salvia MA, Sgarra L, Collino M, \& Montagnani M (2017). Targeting endothelial metaflammation to counteract diabesity cardiovascular risk: Current and perspective therapeutic options. 
Pharmacol Res 120: 226-241.

Powell N, Lo JW, Biancheri P, Vossenkamper A, Pantazi E, Walker AW, et al. (2015). Interleukin 6 Increases Production of Cytokines by Colonic Innate Lymphoid Cells in Mice and Patients With Chronic Intestinal Inflammation. Gastroenterology 149: 456-467 e415.

Ratajczak MZ, \& Kucia M (2020). SARS-CoV-2 infection and overactivation of Nlrp3 inflammasome as a trigger of cytokine "storm" and risk factor for damage of hematopoietic stem cells. Leukemia.

Rayamajhi M, Zhang Y, \& Miao EA (2013). Detection of pyroptosis by measuring released lactate dehydrogenase activity. Methods Mol Biol 1040: 85-90.

Ruan Q, Yang K, Wang W, Jiang L, \& Song J (2020). Clinical predictors of mortality due to COVID-19 based on an analysis of data of 150 patients from Wuhan, China. Intensive Care Med 46(5): 846-848.

Sarzi-Puttini P, Giorgi V, Sirotti S, Marotto D, Ardizzone S, Rizzardini G, et al. (2020). COVID-19, cytokines and immunosuppression: what can we learn from severe acute respiratory syndrome? Clin Exp Rheumatol 38: 337-342.

Serhan CN, Chiang N, \& Dalli J (2015). The resolution code of acute inflammation: Novel pro-resolving lipid mediators in resolution. Semin Immunol 27: 200-215.

Simonnet A, Chetboun M, Poissy J, Raverdy V, Noulette J, Duhamel A, et al. (2020). Lille Intensive Care COVID-19 and Obesity study group. High prevalence of obesity in severe acute respiratory syndrome coronavirus-2 (SARS-CoV-2) requiring invasive mechanical ventilation. . Obesity (Silver Spring).

Singh AK, Gupta R, Ghosh A, \& Misra A (2020). Diabetes in COVID-19: Prevalence, pathophysiology, prognosis and practical considerations. Diabetes Metab Syndr 14: 303-310.

Sodhi M, \& Etminan M (2020). Therapeutic Potential for Tetracyclines in the Treatment of COVID-19. Pharmacotherapy 40: 487-488.

Sun NN, Yu CH, Pan MX, Zhang Y, Zheng BJ, Yang QJ, et al. (2017). Mir-21 Mediates the Inhibitory Effect of Ang (1-7) on AngII-induced NLRP3 Inflammasome Activation by Targeting Spry1 in lung fibroblasts. Sci Rep 7: 14369 .

Tack CJ, Stienstra R, Joosten LA, \& Netea MG (2012). Inflammation links excess fat to insulin resistance: the role of the interleukin-1 family. Immunol Rev 249: 239-252.

Tang TT, Lv LL, Pan MM, Wen Y, Wang B, Li ZL, et al. (2018). Hydroxychloroquine attenuates renal ischemia/reperfusion injury by inhibiting cathepsin mediated NLRP3 inflammasome activation. Cell Death Dis 9: 351 .

Tschop MH, \& DiMarchi RD (2012). Outstanding Scientific Achievement Award Lecture 2011: defeating diabesity: the case for personalized combinatorial therapies. Diabetes 61: 1309-1314.

Vandanmagsar B, Youm YH, Ravussin A, Galgani JE, Stadler K, Mynatt RL, et al. (2011). The NLRP3 inflammasome instigates obesity-induced inflammation and insulin resistance. Nat Med 17: 179-188.

Wang Y, Liu X, Shi H, Yu Y, Yu Y, Li M, et al. (2020). NLRP3 inflammasome, an immune-inflammatory target in pathogenesis and treatment of cardiovascular diseases. Clin Transl Med 10:91-106.

Wanjalla CN, McDonnell WJ, Barnett L, Simmons JD, Furch BD, Lima MC, et al. (2019). Adipose Tissue in Persons With HIV Is Enriched for CD4(+) T Effector Memory and T Effector Memory RA(+) Cells, Which Show Higher CD69 Expression and CD57, CX3CR1, GPR56 Co-expression With Increasing Glucose Intolerance. Front Immunol 10: 408.

Wolowczuk I, Verwaerde C, Viltart O, Delanoye A, Delacre M, Pot B, et al. (2008). Feeding our immune system: impact on metabolism. Clin Dev Immunol 2008: 639803. 
Wu R, Liu X, Yin J, Wu H, Cai X, Wang N, et al. (2018). IL-6 receptor blockade ameliorates diabetic nephropathy via inhibiting inflammasome in mice. Metabolism 83: 18-24.

Xu Y, Ma LL, Zhou C, Zhang FJ, Kong FJ, Wang WN, et al. (2013). Hypercholesterolemic myocardium is vulnerable to ischemia-reperfusion injury and refractory to sevoflurane-induced protection. PLoS One 8: e76652.

Xu Z, Shi L, Wang Y, Zhang J, Huang L, Zhang C, et al. (2020). Pathological findings of COVID-19 associated with acute respiratory distress syndrome. Lancet Respir Med 8: 420-422.

Yan R, Zhang Y, Li Y, Xia L, Guo Y, \& Zhou Q (2020). Structural basis for the recognition of SARS-CoV-2 by full-length human ACE2. Science 367: 1444-1448.

Yang X, Yu Y, Xu J, Shu H, Xia J, Liu H, et al. (2020). Clinical course and outcomes of critically ill patients with SARS-CoV-2 pneumonia in Wuhan, China: a single-centered, retrospective, observational study. Lancet Respir Med 8: 475-481.

Yang Y, Wang H, Kouadir M, Song H, \& Shi F (2019). Recent advances in the mechanisms of NLRP3 inflammasome activation and its inhibitors. Cell Death Dis 10: 128.

Yap JKY, Moriyama M, \& Iwasaki A (2020). Inflammasomes and Pyroptosis as Therapeutic Targets for COVID-19. J Immunol.

Zhang X, Zhang G, Ren Y, Lan T, Li D, Tian J, et al. (2018). Darunavir alleviates irinotecan-induced intestinal toxicity in Vivo. Eur J Pharmacol 834: 288-294.

Zhao C, \& Zhao W (2020). NLRP3 Inflammasome-A Key Player in Antiviral Responses. Front Immunol 11: 211 .

Zhao M, Bai M, Ding G, Zhang Y, Huang S, Jia Z, et al. (2018). Angiotensin II Stimulates the NLRP3 Inflammasome to Induce Podocyte Injury and Mitochondrial Dysfunction. Kidney Dis (Basel) 4:83-94.

Zhou F, Yu T, Du R, Fan G, Liu Y, Liu Z, et al. (2020). Clinical course and risk factors for mortality of adult inpatients with COVID-19 in Wuhan, China: a retrospective cohort study. Lancet 395:1054-1062.

Zuurbier C, Abbate A, Cabrera-Fuentes H, Cohen M, Collino M, De Kleijn D, et al. (2019). Innate immunity as a target for acute cardioprotection. . Cardiovasc Res 115: 1131-1142. 

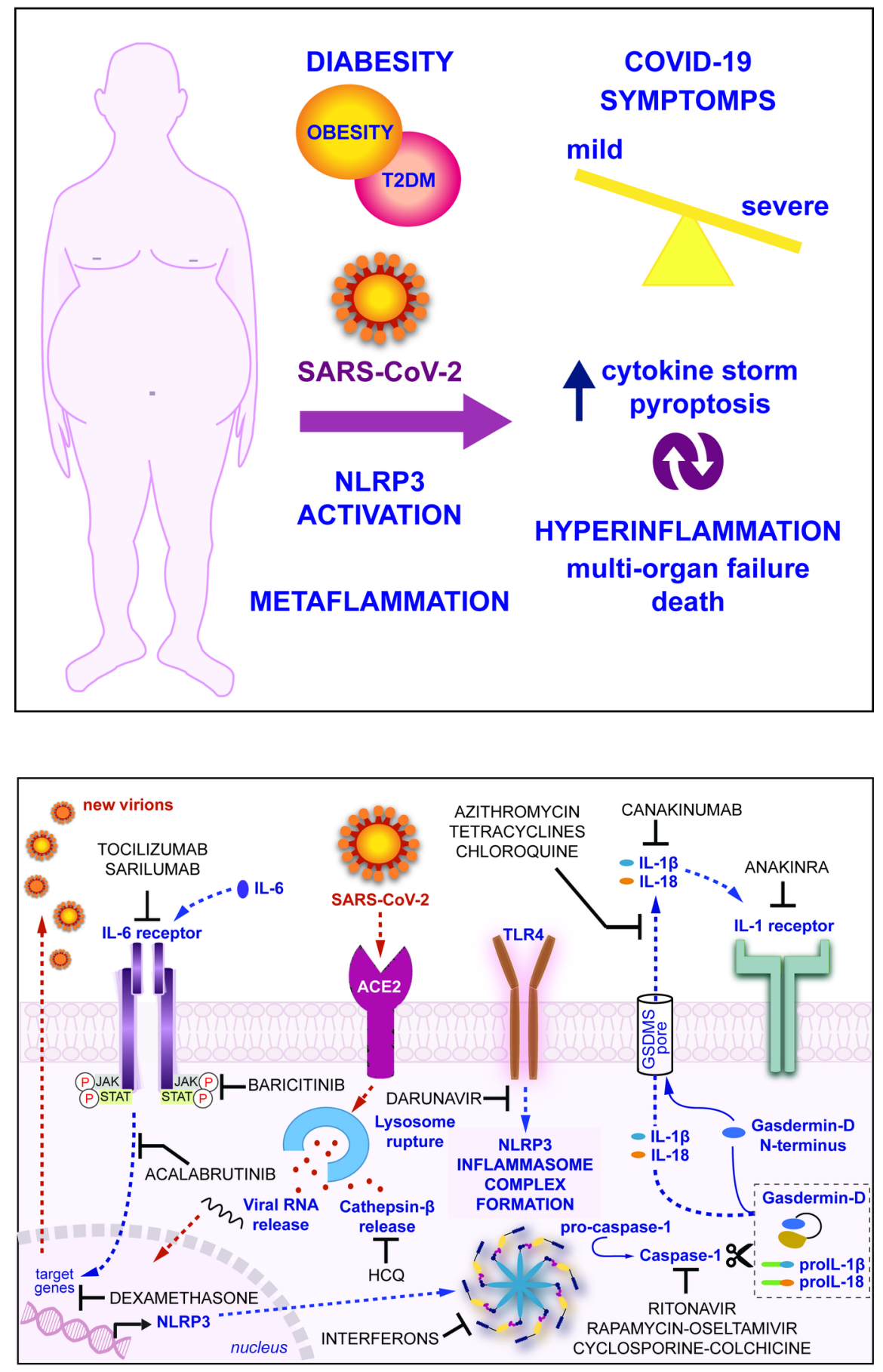